\title{
The Analysis of Distributed Systems with Nonlocal Damping
}

\author{
Yongjun Lei ${ }^{a}$, Michael I. Friswell ${ }^{b}$ and Sondipon Adhikari ${ }^{b}$ \\ ${ }^{a}$ College of Aerospace and Material Engineering, National University of Defense Technology, \\ PR China; \\ ${ }^{b}$ Department of Aerospace Engineering, University of Bristol, Bristol BS8 1TR, UK
}

\begin{abstract}
This paper considers the analysis of structures with nonlocal damping, where the reaction force at any point is obtained as a weighted average of state variables over a spatial domain. The model yields an integro-differential equation, and obtaining closed form solutions is only possible for a limited range of boundary conditions by the transfer function method. Approximate solutions using the Galerkin method for beams are presented for typical spatial kernel functions, for a nonlocal viscoelastic foundation model. This requires the approximation of the displacement to be defined over the whole domain. To treat more complicated problems with variable damping parameters, non-uniform section properties, intermediate supports or arbitrary boundary conditions, a finite element method for beams is developed. However, in nonlocal damping models, nodes remote from the element do have an effect on the energy expressions, and hence the damping matrix is no longer block diagonal. The expressions for these direct and cross damping matrices are obtained for separable spatial kernel functions. The approach is demonstrated on a range of examples.
\end{abstract}

Keywords: Nonlocal damping, Galerkin, Finite elements, Beams, Plates

\section{INTRODUCTION}

The dynamic response analysis of damped structures is important in many areas of mechanical, civil and aerospace engineering, such as the vibration isolation of precise equipment, aircraft noise, or the vibration of cable stayed bridges. Although the damping model plays a key role in the dynamic analysis, particularly for complex structures, this model is often approximated by classical or proportional damping distributions for convenience. In many practical situations this simplified approach does not describe the dynamics of the structure with sufficient accuracy because of the complicated damping mechanisms that occur in practice. Theoretically speaking, any model that makes the energy dissipation function non-negative is a possible candidate for a valid damping model. Research into the dynamics of structures with viscoelastic materials has concentrated on the time dependence using fractional derivative, GHM and other models.

A key issue in considering non-viscous damping is to decide on an appropriate damping model. There have been detailed studies of material damping and damping of specific structural components. Lazan, ${ }^{1}$ Bert ${ }^{2}$ and Ungar $^{3}$ have given excellent accounts of different mathematical methods for modelling damping in a (solid) material and their engineering applications. The book by Nashif et al. ${ }^{4}$ presents more recent studies in this

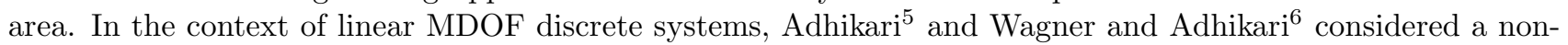
viscous damping model in which the damping forces are assumed to depend on velocity time histories as well as instantaneous velocities. In the context of distributed parameter systems, Banks and Inman ${ }^{7}$ considered nonviscous damping models in Euler-Bernoulli beams. They considered four different models of damping: viscous air damping, Kelvin-Voigt damping, time hysteresis damping and spatial hysteresis damping, and used a spline inverse procedure to form a least-squares fit to the experimental data. It was observed that the spatial hysteresis model combined with a viscous air damping model gave the best quantitative agreement with the experimental time histories. This study motivates us to consider general damping models in distributed parameter systems.

Further author information: (Send correspondence to M.I.F.)

Y.L.: E-mail: leiyj108@yahoo.com.cn

M.I.F.: E-mail: m.i.friswell@bristol.ac.uk, Telephone: +44 (0)117 9288695

S.A.: E-mail: s.adhikari@bristol.ac.uk, Telephone: +44 (0)117 9287694 
This paper considers the effect of nonlocal damping on the response of a structure. In a nonlocal model, the reaction force at any point is obtained as a weighted average of state variables over a spatial domain via convolution integrals with spatial kernel functions that depend on a distance measure. In physical terms the nonlocal damping or elasticity property often arises when two dimensional structures are modelled as one dimensional.

\section{LINEAR DAMPING MODELS FOR DISTRIBUTED PARAMETER SYSTEM}

A linear damped continuous dynamic system in which the displacement vector $\mathbf{u}(\mathbf{r}, t)$, where $\mathbf{r}$ is the spatial position vector and $t$ is time, specified in some domain $\mathcal{D}$, is governed by a linear partial differential equation

$$
\rho(\mathbf{r}) \ddot{\mathbf{u}}(\mathbf{r}, t)+\left(\mathcal{L}_{e}+\mathcal{L}_{i}\right) \dot{\mathbf{u}}(\mathbf{r}, t)+\mathcal{L}_{k} \mathbf{u}(\mathbf{r}, t)=\mathbf{p}(\mathbf{r}, t) ; \quad \mathbf{r} \in \mathcal{D}, \quad t \in[0, T]
$$

with homogeneous linear boundary conditions of the form

$$
\mathcal{M}_{1} \mathbf{u}(\mathbf{r}, t)=\mathbf{g}_{1}(\mathbf{r}, t) ; \quad \mathbf{r} \in \Gamma_{1} \quad \text { and } \quad \mathcal{M}_{2} \dot{\mathbf{u}}(\mathbf{r}, t)=\mathbf{g}_{2}(\mathbf{r}, t) ; \quad \mathbf{r} \in \Gamma_{2}
$$

specified on some boundary surfaces $\Gamma_{1}$ and $\Gamma_{2}$, for given functions $\mathbf{g}_{1}(\mathbf{r}, t)$ and $\mathbf{g}_{2}(\mathbf{r}, t)$.

In the above equation $\rho(\mathbf{r})$ is the mass distribution of the system, $\mathbf{p}(\mathbf{r}, t)$ is the distributed time-varying forcing function, $\mathcal{L}_{k}$ is the spatial self-adjoint stiffness operator and $\mathcal{M}_{1}$ and $\mathcal{M}_{2}$ are linear operators acting on the boundary. $\mathcal{L}_{e}$ and $\mathcal{L}_{i}$ are the external and internal damping operators, respectively, and can be written in the form

$$
\begin{aligned}
\mathcal{L}_{e} \dot{\mathbf{u}}(\mathbf{r}, t) & =\int_{\mathcal{D}} \int_{-\infty}^{t} C_{e}(\mathbf{r}, \boldsymbol{\xi}, t-\tau) \dot{\mathbf{u}}(\boldsymbol{\xi}, \tau) d \tau d \boldsymbol{\xi} \\
\mathcal{L}_{i} \dot{\mathbf{u}}(\mathbf{r}, t) & =\int_{\mathcal{D}} \int_{-\infty}^{t} C_{i}(\mathbf{r}, \boldsymbol{\xi}, t-\tau) \mathcal{L}_{s} \dot{\mathbf{u}}(\boldsymbol{\xi}, \tau) d \tau d \boldsymbol{\xi}
\end{aligned}
$$

where $C_{e}(\mathbf{r}, \boldsymbol{\xi}, t)$ and $C_{e}(\mathbf{r}, \boldsymbol{\xi}, t)$ are the kernel functions for the external and internal damping. The internal and external damping are treated differently because the external damping is only dependent on the displacement, whereas the internal damping is dependent on the internal strains, which are given by spatial derivatives of the displacement through the operator $\mathcal{L}_{s}$. The stiffness operator in Eq. (1), $\mathcal{L}_{k}$, may be treated in a similar way when the distributed parameter dynamic system has nonlocal elastic or viscoelastic material. In this paper we only consider the nonlocal influence of the damping operators, $\mathcal{L}_{e}$ and $\mathcal{L}_{i}$, although the extension to nonlocal stiffness is straight-forward. The velocities $\dot{\mathbf{u}}(\boldsymbol{\xi}, \tau)$ at different time instants and spatial locations are coupled through this kernel function. Kernel functions that serve similar purposes have been described by different names in different subjects (for example, retardation functions, heredity functions, after-effect functions, relaxation functions), and different models have been used to describe them. In principle, any function that makes the rate of energy dissipation (given here for external damping only),

$$
\mathcal{F}(t)=\frac{1}{2} \int_{\mathcal{D}}\left\{\int_{\mathcal{D}} \int_{-\infty}^{t} C_{e}(\mathbf{r}, \boldsymbol{\xi}, t-\tau) \dot{\mathbf{u}}(\boldsymbol{\xi}, \tau) d \tau d \boldsymbol{\xi}\right\} \dot{\mathbf{u}}(\mathbf{r}, t) d \mathbf{r}
$$

non-negative can be used as a kernel function. Equation (1) together with Eqs. (3) and (4) represents a continuous dynamic system with general linear damping. It may be noted that if $\mathcal{L}_{e}=\mathcal{L}_{i}=0$ in Eq. (1), i.e. an undamped system, or if the system satisfies the criteria given by Caughey and O'Kelly, ${ }^{8}$ then the system will possess classical normal modes. However, due to the general nature of the operators $\mathcal{L}_{e}$ and $\mathcal{L}_{i}$ there is no definite reason why the system should have classical normal modes. Thus, in general, the mode shapes and natural frequencies of such systems will be complex in nature. Note that the system expressed by Eq. (1) and the damping operators defined in Eqs. (3) and (4) represents a partial integro-differential equation with the boundary conditions given in Eq. (2). In this paper we are interested in the natural frequencies and mode shapes of the system. Exact closed-form expressions of such quantities for the general case are difficult to obtain. We make the following general assumptions:

- the mass and stiffness distributions are homogeneous, that is, they do not vary with the position vector $\mathbf{r}$, and 
- the damping kernel functions $C_{e}(\mathbf{r}, \boldsymbol{\xi}, t)$ and $C_{i}(\mathbf{r}, \boldsymbol{\xi}, t)$ are separable in space and time.

If the kernel function is separable in space and time, then either kernel function takes form

$$
C(\mathbf{r}, \boldsymbol{\xi}, t-\tau)=H(\mathbf{r}) c(\mathbf{r}-\boldsymbol{\xi}) g(t-\tau) .
$$

Note the subscripts $e$ and $i$ have been dropped from the kernel function, and either externel or internal damping kernels may be written in the form given in Eq. (6). Physically this model represents nonlocal viscoelastic damping, for example, in a beam with viscoelastic material or an actively controlled piezoelectric patch. Viscous damping has the form $g(t-\tau)=\delta(t-\tau)$, and local damping $c(\mathbf{r}-\boldsymbol{\xi})=\delta(\mathbf{r}-\boldsymbol{\xi})$.

The following functional forms for the kernel functions are common, and hence chosen to study further,

$$
\begin{aligned}
g(t) & =g_{\infty} \mu \exp (-\mu t) \quad \text { so that } \quad G(s)=\frac{g_{\infty} \mu}{s+\mu} \\
\text { and } \quad c(\mathbf{r}-\boldsymbol{\xi}) & =\frac{\alpha}{2} \exp (-\alpha|\mathbf{r}-\boldsymbol{\xi}|), \quad H(\mathbf{r}) \geq 0,
\end{aligned}
$$

where $g_{\infty}, \mu$ and $\alpha$ are all positive constants. If $\alpha \rightarrow \infty, \mu \rightarrow \infty$ then one obtains the standard local viscous model. If $\alpha \rightarrow \infty$ and $\mu$ is finite one obtains the local non-viscous model and if $\alpha$ is finite but $\mu \rightarrow \infty$ one obtains the nonlocal viscous damping model. Thus, it is expected that the natural frequencies and the mode shapes in these parameter cases of the general model should approach the appropriate special cases.

\section{EQUATION OF MOTION FOR A BEAM}

Suppose that nonlocal damping exists for a beam between locations $x_{1}$ and $x_{2}$, as shown in Fig. 1. These locations could be different for the internal and external damping, but this extension is very straight-forward. The internal damping model is taken as that given Sorrentino et al.. ${ }^{9}$ The equation of motion for this beam may be expressed as the following integro-partial-differential equation,

$$
\begin{aligned}
\frac{\partial^{2}}{\partial x^{2}}\left(E I(x) \frac{\partial^{2} w(x, t)}{\partial x^{2}}\right)+\rho A(x) & \frac{\partial^{2} w(x, t)}{\partial t^{2}}+\int_{x_{1}}^{x_{2}} \int_{-\infty}^{t} C_{e}(x, \xi, t-\tau) \frac{\partial w(\xi, \tau)}{\partial t} d \tau d \xi \\
& +\int_{x_{1}}^{x_{2}} \int_{-\infty}^{t} C_{i}(x, \xi, t-\tau) \frac{\partial^{2}}{\partial \xi^{2}}\left(\gamma(\xi) \frac{\partial^{3} w(\xi, \tau)}{\partial \xi^{2} \partial \tau}\right) d \tau d \xi=f(x, t)
\end{aligned}
$$

where $E I(x)$ is the bending stiffness, $\rho A(x)$ is the mass per unit length, $\gamma(x)$ is the internal damping coefficient, $w(x, t)$ is the transverse displacement, and $f(x, t)$ is the distributed external force. In Eq. (9), the third and fourth terms are the nonlocal external and internal damping. $C_{e}(x, \xi, t-\tau)$ and $C_{i}(x, \xi, t-\tau)$ are the onedimensional kernel functions of the form described in the previous section, defined over the spatial sub domain $\left(x_{1}, x_{2}\right)$. Thus,

$$
c(x-\xi)=\frac{\alpha}{2} e^{-\alpha|x-\xi|} .
$$

Lei et al..$^{10}$ gave examples of other common kernel functions.

The appropriate boundary conditions must be satisfied at $x=0$ and $L$, and typically they are the three simple cases of clamped, simple supported or free.

Taking the Laplace transform of Eq. (9) gives the eigenvalue problem for the free vibration of the beam as

$$
\begin{aligned}
\frac{\partial^{2}}{\partial x^{2}}\left(E I(x) \frac{\partial^{2} W(x, s)}{\partial x^{2}}\right)+s^{2} \rho A(x) W(x, s) & +s G_{e}(s) H_{0} \int_{x_{1}}^{x_{2}} c_{e}(x-\xi) W(\xi, s) d \xi \\
& +s G_{i}(s) \int_{x_{1}}^{x_{2}} c_{i}(x-\xi) \frac{\partial^{2}}{\partial \xi^{2}}\left(\gamma(\xi) \frac{\partial^{2} W(\xi, s)}{\partial \xi^{2}}\right) d \xi=0
\end{aligned}
$$




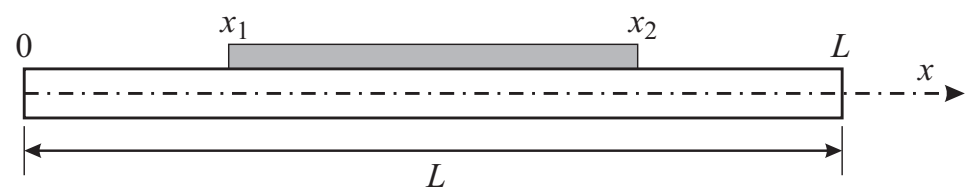

Figure 1. A beam with a partial nonlocal damping patch.

where $G_{e}(s)$ and $G_{i}(s)$ are the Laplace transforms of the external and internal kernel functions $g_{e}(t)$ and $g_{i}(t)$, and $W(x, s)$ is the Laplace transform of $w(x, t)$. Equation (11) is an integro-differential equation, and obtaining closed form solutions is difficult. Approximate solutions may be obtained using the Galerkin method ${ }^{10,11}$ or finite element analysis.

\section{THE GALERKIN SOLUTION FOR A NON-LOCALLY DAMPED BEAM}

In the Galerkin approach, the response is approximated by the series, ${ }^{10,11}$

$$
W(x, s)=\sum_{j=1}^{N} q_{j}(s) \phi_{j}(x)
$$

where $\phi_{j}(x),(j=1,2, \ldots, N)$ are given admissible (or trial) functions satisfying the essential boundary conditions, $N$ is the number of admissible functions and is determined by the accuracy required. The admissible functions $\phi_{j}(x)$ can be chosen in many ways, for example as trigonometric functions, interpolating polynomials, orthogonal polynomials or exponential functions. A good choice for the beam vibration problem, especially with light damping, is to use the undamped mode shapes.

Substituting $W(x, s)$ into Eq. (11), multiplying by $\phi_{k}(x)$ and integrating with respect to $x$ over the length of the beam, gives

$$
\left[s^{2} \mathbf{M}+s G_{i}(s) \mathbf{C}_{i}+s G_{e}(s) \mathbf{C}_{e}+\mathbf{K}\right] \mathbf{q}=0
$$

where $\mathbf{K}, \mathbf{M}, \mathbf{C}_{i}$ and $\mathbf{C}_{e}$ are the mass, stiffness and damping (internal and external) matrices with respect to the generalised co-ordinates $\mathbf{q}=\left\{q_{j}\right\}$. The elements of these matrices are, for free, simply supported or clamped boundary conditions, ${ }^{10}$

$$
\begin{aligned}
M_{k j} & =\int_{0}^{L} \rho A(x) \phi_{j}(x) \phi_{k}(x) d x, \\
C_{e k j} & =H_{0} \int_{x_{1}}^{x_{2}} \int_{x_{1}}^{x_{2}} c_{e}(x-\xi) \phi_{j}(\xi) \phi_{k}(x) d \xi d x, \\
C_{i k j} & =\int_{x_{1}}^{x_{2}} \int_{x_{1}}^{x_{2}} c_{i}(x-\xi) \frac{d^{2}}{d \xi^{2}}\left(\gamma(\xi) \frac{d^{2} \phi_{j}(\xi)}{d \xi^{2}}\right) \phi_{k}(x) d \xi d x, \\
K_{k j} & =\int_{0}^{L} \frac{d^{2}}{d x^{2}}\left(E I(x) \frac{d^{2} \phi_{j}(x)}{d x^{2}}\right) \phi_{k}(x) d x=\int_{0}^{L} E I(x) \frac{d^{2} \phi_{j}(x)}{d x^{2}} \frac{d^{2} \phi_{k}(x)}{d x^{2}} d x .
\end{aligned}
$$

If the undamped modes are used as admissible functions then the mass and stiffness matrices are diagonal. In addition, the modes for beams with simple boundary conditions are trignometric functions, and hence may be written in exponential form to perform the integration required to calculate the damping matrix for the kernel given in Eq. (10).

The eigenvalues are obtained by solving

$$
\operatorname{det}\left|s^{2} \mathbf{M}+s G_{i}(s) \mathbf{C}_{i}+s G_{e}(s) \mathbf{C}_{e}+\mathbf{K}\right|=0 .
$$

The corresponding mode shape functions are obtained by substituting the eigenvalues into Eq. (13) and computing the null space of the matrix. Alternatively, for kernel functions where $G(s)$ is a rational polynomial, the internal variable approach (for example the GHM method ${ }^{12-14}$ ) may be used to produce a linear eigenvalue problem, where the size of the matrices is larger than those in Eq. (15). 


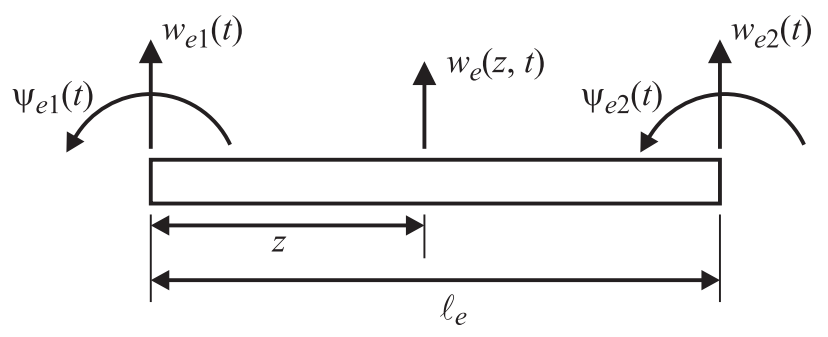

Figure 2. The degrees of freedom of a beam finite element.

\section{FINITE ELEMENT ANALYSIS}

Finite element analysis may be considered as a Galerkin method, where the admissible functions are only defined over a small region of the structure. Generally the approach adopted when developing models using finite element analysis is to approximate the deformation within an element using nodal values of displacement and rotation. The kinetic and strain energy for each element is then computed and the contributions of each element added together to obtain a global model of the structure. One key aspect of finite element analysis is that the energy contributions from each element only depends on the displacements and rotations at the nodes associated with that element. Clearly for nonlocal damping this will not be the case, although the form of the kernel function given by Eq. (10) does lead to considerable simplification. This paper only considers nonlocal damping, and so the development of the mass and stiffness matrices follows the standard procedure, which will be outlined briefly to establish notation.

A standard beam element is modelled using two nodes (at the ends of the beam element), and two degrees of freedom per node (translation and rotation), as shown in Fig. 2. The deformation within the eth element, $w_{e}(z, t)$, is approximated using cubic shape shape functions, for $z \in\left[0, \ell_{e}\right]$, as

$$
w_{e}(z, t)=\mathbf{N}_{e}(z) \mathbf{q}_{e}(t), \quad \text { where } \quad \mathbf{N}_{e}(z)=\left[N_{e 1}(z) N_{e 2}(z) N_{e 3}(z) N_{e 4}(z)\right], \quad \mathbf{q}_{e}(t)=\left\{\begin{array}{l}
w_{e 1}(t) \\
\psi_{e 1}(t) \\
w_{e 2}(t) \\
\psi_{e 2}(t)
\end{array}\right\}
$$

and

$$
\begin{array}{ll}
N_{e 1}(z)=1-3 \frac{z^{2}}{\ell_{e}^{2}}+2 \frac{z^{3}}{\ell_{e}^{3}}, & N_{e 2}(z)=z-2 \frac{z^{2}}{\ell_{e}}+\frac{z^{3}}{\ell^{2}} \\
N_{e 3}(z)=3 \frac{z^{2}}{\ell_{e}^{2}}-2 \frac{z^{3}}{\ell_{e}^{3}}, & N_{e 4}(z)=-\frac{z^{2}}{\ell_{e}}+\frac{z^{3}}{\ell_{e}^{2}} .
\end{array}
$$

The kinetic energy, for a beam with $M$ elements, is then approximated as

$$
T=\frac{1}{2} \int_{0}^{L} \rho A(x)\left(\frac{\partial w(x, t)}{\partial t}\right)^{2} d x=\frac{1}{2} \sum_{e=1}^{M} \int_{0}^{\ell_{e}} \rho A\left(x_{e}+z\right)\left(\frac{\partial w_{e}(z, t)}{\partial t}\right)^{2} d z=\frac{1}{2} \sum_{e=1}^{M} \dot{\mathbf{q}}_{e}(t)^{T} \mathbf{M}_{e} \dot{\mathbf{q}}_{e}(t),
$$

where the $e$ th node, corresponding to node 1 of the eth element, is located at $x_{e}$, and the element mass matrix is

$$
\mathbf{M}_{e}=\int_{0}^{\ell_{e}} \rho A\left(x_{e}+z\right) \mathbf{N}_{e}^{T} \mathbf{N}_{e} d z
$$

For nonlocal damping the global damping matrix is obtained in a similar way using the energy dissipation function. Consider external damping only for the moment. The dissipation function is then

$$
\mathcal{F}(t)=\frac{1}{2} \int_{0}^{L}\left\{\int_{0}^{L} \int_{-\infty}^{t} C_{e}(x, \xi, t-\tau) \dot{w}(\xi, \tau) d \tau d \xi\right\} \dot{w}(x, t) d x
$$


Assuming that the kernel is separable, the order of integration may be changed, and that $H_{e}(x)=1$, then

$$
\begin{aligned}
\mathcal{F}(t) & =\frac{1}{2} \int_{-\infty}^{t} g_{e}(t-\tau)\left\{\int_{0}^{L} \int_{0}^{L} c_{e}(x-\xi) \dot{w}(\xi, \tau) \dot{w}(x, t) d \xi d x\right\} d \tau \\
& =\frac{1}{2} \int_{-\infty}^{t} g_{e}(t-\tau) \sum_{i, j=1}^{M} \dot{\mathbf{q}}_{i}(t)^{T} \mathbf{C}_{i j} \dot{\mathbf{q}}_{j}(\tau) d \tau
\end{aligned}
$$

where

$$
\mathbf{C}_{i j}=\int_{0}^{\ell_{j}} \int_{0}^{\ell_{i}} c_{e}\left(x_{j}+\hat{x}-x_{i}-\hat{\xi}\right) \mathbf{N}_{i}^{T}(\hat{\xi}) \mathbf{N}_{j}(\hat{x}) d \hat{\xi} d \hat{x}
$$

and $\hat{x}$ and $\hat{\xi}$ are local co-ordinates, for example $x=x_{j}+\hat{x}$.

In general the degrees of freedom at all nodes will be coupled within a region where the structure has nonlocal damping behaviour. This means the global damping matrix will be full, and each term must be determined independently. However the exponential kernel function given in Eq. (10) leads to a significant simplification for beams modelled using beam elements of equal length, so that $\mathbf{N}_{i}=\mathbf{N}, \ell_{i}=\ell, \forall i$. When $i=j$, the direct damping matrix is

$$
\mathbf{C}_{i i}=\frac{1}{\alpha} \int_{0}^{\ell} \int_{0}^{\ell} e^{-\alpha|\hat{x}-\hat{\xi}|} \mathbf{N}^{T}(\hat{\xi}) \mathbf{N}(\hat{x}) d \hat{\xi} d \hat{x}
$$

If $j>i$

$$
\mathbf{C}_{i j}=\frac{1}{\alpha} \int_{0}^{\ell} \int_{0}^{\ell} e^{-\alpha\left(x_{j}-x_{i}+\hat{x}-\hat{\xi}\right)} \mathbf{N}^{T}(\hat{\xi}) \mathbf{N}(\hat{x}) d \hat{\xi} d \hat{x}=e^{-\alpha\left(x_{j}-x_{i}\right)} \overline{\mathbf{C}}=e^{-\alpha(j-i) \ell} \overline{\mathbf{C}},
$$

where the cross damping matrix is

$$
\overline{\mathbf{C}}=\frac{1}{\alpha} \int_{0}^{\ell} \int_{0}^{\ell} e^{-\alpha(\hat{x}-\hat{\xi})} \mathbf{N}^{T}(\hat{\xi}) \mathbf{N}(\hat{x}) d \hat{\xi} d \hat{x}=\frac{1}{\alpha}\left(\int_{0}^{\ell} e^{\alpha \hat{\xi}} \mathbf{N}^{T}(\hat{\xi}) d \hat{\xi}\right)\left(\int_{0}^{\ell} e^{-\alpha \hat{x}} \mathbf{N}(\hat{x}) d \hat{x}\right) .
$$

Similarly if $j<i$,

$$
\mathbf{C}_{i j}=e^{-\alpha(i-j) \ell} \overline{\mathbf{C}}^{T}
$$

Thus only two element damping matrices need to be computed and then the complete global damping matrix is easily derived. These matrices may be obtained explicitly by integrating Eqs. (23) or (25). Alternatively they may be derived by numerical integration.

As an example to demonstrate how the damping matrix is assembled, suppose that a uniform beam is modelled, where three elements have a nonlocal damped foundation. Then the damping matrix will be zero apart from the eight degrees of freedom associated with the damped foundation, and the non-zero terms are

$$
\begin{aligned}
\mathbf{C}= & {\left[\begin{array}{ll}
\mathbf{C}_{i i} & \mathbf{0}_{44} \\
\mathbf{0}_{44} & \mathbf{0}_{44}
\end{array}\right]+\left[\begin{array}{lll}
\mathbf{0}_{22} & \mathbf{0}_{24} & \mathbf{0}_{22} \\
\mathbf{0}_{42} & \mathbf{C}_{i i} & \mathbf{0}_{42} \\
\mathbf{0}_{22} & \mathbf{0}_{24} & \mathbf{0}_{22}
\end{array}\right]+\left[\begin{array}{ll}
\mathbf{0}_{44} & \mathbf{0}_{44} \\
\mathbf{0}_{44} & \mathbf{C}_{i i}
\end{array}\right] } \\
& +e^{-\alpha \ell}\left\{\left[\begin{array}{lll}
\mathbf{0}_{42} & \overline{\mathbf{C}} & \mathbf{0}_{42} \\
\mathbf{0}_{42} & \mathbf{0}_{44} & \mathbf{0}_{42}
\end{array}\right]+\left[\begin{array}{cc}
\mathbf{0}_{24} & \mathbf{0}_{24} \\
\mathbf{0}_{44} & \overline{\mathbf{C}} \\
\mathbf{0}_{24} & \mathbf{0}_{24}
\end{array}\right]+\left[\begin{array}{ll}
\mathbf{0}_{24} & \mathbf{0}_{24} \\
\overline{\mathbf{C}}^{T} & \mathbf{0}_{44} \\
\mathbf{0}_{24} & \mathbf{0}_{24}
\end{array}\right]+\left[\begin{array}{lll}
\mathbf{0}_{42} & \mathbf{0}_{44} & \mathbf{0}_{42} \\
\mathbf{0}_{42} & \overline{\mathbf{C}}^{T} & \mathbf{0}_{42}
\end{array}\right]\right\} \\
& +e^{-2 \alpha \ell}\left\{\left[\begin{array}{ll}
\mathbf{0}_{44} & \overline{\mathbf{C}} \\
\mathbf{0}_{44} & \mathbf{0}_{44}
\end{array}\right]+\left[\begin{array}{ll}
\mathbf{0}_{44} & \mathbf{0}_{44} \\
\overline{\mathbf{C}}^{T} & \mathbf{0}_{44}
\end{array}\right]\right\}
\end{aligned}
$$

where $\mathbf{0}_{r s}$ is the $r \times s$ zero matrix. 


\section{EXAMPLE}

A pinned-pinned beam will be used as an example to compare the Galerkin and finite element methods. In this case the $i$ th mode of the undamped beam is $\phi_{i}(x)=A_{i} \sin (i \pi x / L)$ where $A_{i}$ is calculated to give mass normalised mode shapes. These undamped mode shapes will be used as the admissible functions in the Galerkin approach. The nonlocal damping layer is assumed to be external, which models a damped foundation. Initially the dimensions are (see Fig. 1) $L=200 \mathrm{~mm}, x_{1}=50 \mathrm{~mm}$, and $x_{2}=150 \mathrm{~mm}$. The Young's modulus is $E=$ $70 \mathrm{GN} / \mathrm{m}^{2}$, the density is $\rho=2700 \mathrm{~kg} / \mathrm{m}^{3}$, and the cross-section is $5 \times 5 \mathrm{~mm}$.

Table 1 shows the first 3 eigenvalue pairs for the pinned-pinned beam example with viscous damping $(\mu \rightarrow \infty)$, $\alpha=1, g_{\infty}=1$ and $H=200$ for the Galerkin method with 10 admissible functions and also for the finite element method with 4,8 and 40 elements.

Table 2 shows the first 3 eigenvalue pairs for the pinned-pinned beam example with a variety of non-viscous and nonlocal damping, that is as $\mu$ and $\alpha$ vary, for the Galerkin method with 10 admissible functions.

Suppose now that the beam is clamped at one end and free at the other. All other dimensions are identical to the pinned-pinned beam example. Table 3 shows the first 3 eigenvalue pairs for this cantilever beam example with a variety of non-viscous and nonlocal damping, that is as $\mu$ and $\alpha$ vary, for the finite element method with 8 elements. Clearly both the nonlocal and non-viscous properties of the beam have a significant effect on the damping within the structure.

Table 1. The first 3 eigenvalue pairs for the pinned-pinned beam example obtain from the Galerkin method with 10 admissible functions and the finite element (FE) method with 4,8 and 40 elements. $\alpha=1, g_{\infty}=1, H=200, \mu \rightarrow \infty$

\begin{tabular}{|c|c|c|c|}
\hline Galerkin & FE (4 elements) & FE (8 elements) & FE (40 elements $)$ \\
\hline$-58.176 \pm 1812.4 \mathrm{j}$ & $-58.174 \pm 1812.9 \mathrm{j}$ & $-58.176 \pm 1812.5 \mathrm{j}$ & $-58.176 \pm 1812.4 \mathrm{j}$ \\
\hline$-0.72086 \pm 7253.5 \mathrm{j}$ & $-0.72080 \pm 7282.1 \mathrm{j}$ & $-0.72086 \pm 7255.4 \mathrm{j}$ & $-0.72086 \pm 7253.5 \mathrm{j}$ \\
\hline$-6.7383 \pm 16320 \mathrm{j}$ & $-6.5458 \pm 16618 \mathrm{j}$ & $-6.7359 \pm 16341 \mathrm{j}$ & $-6.7384 \pm 16320 \mathrm{j}$ \\
\hline
\end{tabular}

Table 2. The first 3 eigenvalue pairs for the pinned-pinned beam example obtained from the Galerkin method with 10 admissible functions for different values of $\mu$ and $\alpha$.

\begin{tabular}{|c|c|c|c|c|}
\hline$\mu$ & $\infty$ & 1000 & 1000 & $\infty$ \\
\hline$\alpha$ & 1 & 1 & 10 & 10 \\
\hline & $-58.176 \pm 1812.4 \mathrm{j}$ & $-13.367 \pm 1838.1 \mathrm{j}$ & $-92.218 \pm 2006.0 \mathrm{j}$ & $-447.62 \pm 1757.7 \mathrm{j}$ \\
\hline & $-0.72086 \pm 7253.5 \mathrm{j}$ & $-0.013445 \pm 7253.6 \mathrm{j}$ & $-0.94926 \pm 7260.4 \mathrm{j}$ & $-50.996 \pm 7253.3 \mathrm{j}$ \\
\hline & $-6.7383 \pm 16320 \mathrm{j}$ & $-0.025225 \pm 16321 \mathrm{j}$ & $-0.26635 \pm 16325 \mathrm{j}$ & $-70.636 \pm 16317 \mathrm{j}$ \\
\hline
\end{tabular}

\section{CONCLUSIONS}

This paper has introduced two approximate methods to estimate the eigenvalues of structures with nonlocal and non-viscous damping. The Galerkin method may be applied if suitable admissible functions can be found to approximate the displacement within the structure and also satisfy the essential boundary conditions. However the finite element approach is more widely applicable to general structures. Although the nonlocal damping properties cause a coupling between degrees of freedom not normally coupled in the finite element analysis, the global damping matrix for a uniform beam with an exponential damping kernel only requires the calculation of two element matrices. The approaches have been demonstrated on pinned-pinned and cantilever beam examples, and the convergence of the finite element model, and the effect of nonlocal and non-viscous properties, have been highlighted. 
Table 3. The first 3 eigenvalue pairs for the cantilever beam example obtained from the finite element method with 8 elements for different values of $\mu$ and $\alpha$.

\begin{tabular}{|c|c|c|c|c|}
\hline$\mu$ & $\infty$ & 1000 & 1000 & $\infty$ \\
\hline$\alpha$ & 1 & 1 & 10 & 10 \\
\hline & $-17.841 \pm 645.83 \mathrm{j}$ & $-12.672 \pm 654.28 \mathrm{j}$ & $-102.77 \pm 721.46 \mathrm{j}$ & $-141.58 \pm 634.22 \mathrm{j}$ \\
\hline & $-45.254 \pm 4048.1 \mathrm{j}$ & $-2.6009 \pm 4059.3 \mathrm{j}$ & $-20.277 \pm 4132.1 \mathrm{j}$ & $-353.66 \pm 4009.6 \mathrm{j}$ \\
\hline & $-1.0206 \pm 11343 \mathrm{j}$ & $-0.007875 \pm 11343 \mathrm{j}$ & $-0.47410 \pm 11348 \mathrm{j}$ & $-61.492 \pm 11342 \mathrm{j}$ \\
\hline
\end{tabular}

\section{ACKNOWLEDGMENTS}

Yongjun Lei gratefully acknowledges the support of China Scholarship Council through a Scholarship Fund Award. Michael Friswell gratefully acknowledges the support of the Royal Society through a Royal SocietyWolfson Research Merit Award. Sondipon Adhikari gratefully acknowledges the support of the Engineering and Physical Sciences Research Council through the award of an Advanced Research Fellowship.

\section{REFERENCES}

1. B. J. Lazan, Damping of Materials and Members in Structural Mechanics, Pergamon Press, Oxford, 1968.

2. C. W. Bert, "Material damping: an introductory review of mathematical models, measure and experimental techniques," Journal of Sound and Vibration 29(2), pp. 129-153, 1973.

3. E. E. Ungar, "The status of engineering knowledge concerning the damping of built-up structures," Journal of Sound and Vibration 26, pp. 141-154, 1973.

4. A. D. Nashif, D. I. G. Jones, and J. P. Henderson, Vibration Damping, John Wiley, New York, 1985.

5. S. Adhikari, "Dynamics of non-viscously damped linear systems," ASCE Journal of Engineering Mechanics 128, pp. 328-339, March 2002.

6. N. Wagner and S. Adhikari, "Symmetric state-space formulation for a class of non-viscously damped systems," AIAA Journal 41(5), pp. 951-956, 2003.

7. H. T. Banks and D. J. Inman, "On damping mechanisms in beams," Transactions of ASME, Journal of Applied Mechanics 58, pp. 716-723, September 1991.

8. T. K. Caughey and M. E. J. O'Kelly, "Classical normal modes in damped linear dynamic systems," Transactions of ASME, Journal of Applied Mechanics 32, pp. 583-588, September 1965.

9. S. Sorrentino, S. Marchesiello, and B. Piombo, "A new analytical technique for vibration analysis of nonproportionally damped beams," Journal of Sound and Vibration 265, pp. 765-782, 2003.

10. Y. Lei, M. I. Friswell, and S. Adhikari, "A Galerkin method for distributed systems with non-local damping," International Journal of Solids and Structures 43, p. in press, 2006.

11. C. A. J. Fletcher, Computational Galerkin Methods, Springer-Verlag, New York, 1984.

12. D. F. Golla and P. C. Hughes, "Dynamics of viscoelastic structures - a time domain finite element formulation," Transactions of ASME, Journal of Applied Mechanics 52, pp. 897-906, December 1985.

13. D. J. McTavish and P. C. Hughes, "Modeling of linear viscoelastic space structures," Transactions of ASME, Journal of Vibration and Acoustics 115, pp. 103-110, January 1993.

14. M. I. Friswell, D. J. Inman, and M. J. Lam, "On the realisation of ghm models in viscoelasticity," Journal of Intelligent Material Systems and Structures 8, pp. 986-993, November 1997. 